\title{
BAND MIXING EFFECTS IN QUANTUM WELL MAGNETOEXCITONS
}

\author{
R. WYSOCKI, W. BARDYSZEWSKI \\ Institute of Theoretical Physics, Warsaw University, Hoża 69, 00-681 Warsaw, Poland
}

\section{S. Schoser AND M. PotemsKi}

\author{
Grenoble High Magnetic Field Laboratory, MPI/FKF and CNRS \\ BP 166 Grenoble, Grenoble cedex 9, France
}

\begin{abstract}
The influence of intersubband mixing in quantum wells of semiconductors with zinc-blende structure is studied both experimentally and theoretically. A multiband magnetoexciton model is described which takes into account $k \cdot p$ mixing between valence subbands and the effective Coulomb interaction for an arbitrary confinement potential shape. Theoretical results reproduce very well the photoluminescence excitation spectra of GaAs/AlGaAs single quantum wells of various widths. In particular, the characteristic avoided crossing between the lowest light-hole exciton Landau level and excited heavy-hole exciton Landau level occurring at $\sigma^{-}$polarization is accurately described by our theory.
\end{abstract}

PACS numbers: 71.35.Ji, 71.70.Ej

\section{Introduction}

The characteristic subband splitting in typical quantum wells is comparable to the exciton binding energy. Coulomb interaction between electrons and holes leads to the confinement of motion of each constituent of an exciton to a volume characterized by the exciton radius. Applying external magnetic field introduces another characteristic length scale, the so-called magnetic length and the associated energy - the cyclotron frequency. A very intricate energy spectrum may result when the three lengths: well width, exciton radius and the magnetic length are comparable, as a result of mixing between the light and heavy hole subbands [1]. In particular, we will show that the additional confinement of electron and hole wave functions caused by the external magnetic field enhances the $k \cdot p$ mixing leading to the characteristic avoided crossings between subbands which are clearly distinguishable at higher fields for narrower quantum wells. 


\section{Theory}

The interband absorption coefficient can be expressed in terms of the electron-hole pair correlation function [2]:

$$
\Pi_{12}(t)=\Theta(t)\left\langle\left[a_{2}^{\dagger}(t) a_{1}(t), \hat{P}_{\alpha}^{\dagger}(0)\right]_{-}\right\rangle
$$

where $a_{2}^{\dagger}(t)$ and $a_{1}(t)$ denote the creation and annihilation operators in the single-particle states 2 belonging to the group of valence subbands and the conduction band states labeled by the index 1 . The initial state of an optically excited electron-hole pair is created by the interband part of the momentum operator between the valence and conduction subbands which for a polarization of light $\alpha$ is given by

$$
\widehat{\boldsymbol{P}}_{\alpha}^{\dagger}(t)=\sum_{12}\left\langle 1\left|\hat{\boldsymbol{p}}^{\alpha}(t)\right| 2\right\rangle \mathrm{a}_{1}^{\dagger}(t) \mathrm{a}_{2}(t)=\sum_{12} P_{12}^{\alpha} \mathrm{a}_{1}^{\dagger}(t) \mathrm{a}_{2}(t) .
$$

The absorption coefficient is then proportional to the spectral density function for the exciton autocorrelation function

$$
\alpha(\omega)=\frac{4 \pi}{c n_{r} \omega V} \operatorname{Im}\left[\mathrm{i} \sum_{12} P_{21}^{\alpha} \int_{0}^{\infty} \Pi_{12}(t) \mathrm{e}^{\mathrm{i}(\omega+\mathrm{i} \eta) t} \mathrm{~d} t\right] .
$$

The central problem of our theory is to solve the approximate equation of motion for the excitonic wave function in the presence of the magnetic field and confinement potential of the well

$$
\begin{aligned}
& \mathrm{i} \hbar \partial_{t} \Pi_{12}(t)=\mathrm{i} \hbar \delta(t) \sum_{1^{\prime} 2^{\prime}}\left(\rho_{2^{\prime} 2}^{\mathrm{v}} \delta_{11^{\prime}}-\rho^{\mathrm{c}}{ }_{11^{\prime}} \delta_{2^{\prime} 2}\right) P_{1^{\prime} 2^{\prime}}^{\alpha}+\sum_{1^{\prime} 2^{\prime}}\left(H^{\mathrm{c}}{ }_{11^{\prime}} \delta_{2^{\prime} 2}-H_{2^{\prime} 2}^{\mathrm{v}} \delta_{11^{\prime}}\right) \\
& \times \Pi_{1^{\prime} 2^{\prime}}(t)+\sum_{1^{\prime} 2^{\prime} 1^{\prime \prime} 2^{\prime \prime}}\left(\rho_{11^{\prime \prime}}^{\mathrm{c}} \delta_{2^{\prime \prime 2}}-\rho_{2^{\prime \prime}{ }^{\prime}}^{\mathrm{v}} \delta_{11^{\prime \prime}}\right) U_{1^{\prime \prime} 2^{\prime} 2^{\prime \prime} 1^{\prime}} \Pi_{1^{\prime} 2^{\prime}}(t)
\end{aligned}
$$

where $H_{11^{\prime}}^{\mathrm{c}}, \rho_{11^{\prime}}^{\mathrm{c}}$, and $H_{2^{\prime 2}}^{v}, \rho_{2^{\prime} 2}^{v}$, denote the matrix elements of the single-particle Hamiltonians and single-particle density matrices corresponding to the states in

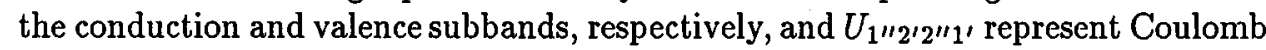
interaction matrix elements. In our two-dimensional $k \cdot p$ approach we have obtained the kinetic part of the valence band matrix $H_{2^{\prime 2}}^{\mathrm{v}}$ from the corresponding three-dimensional Luttinger-Kohn Hamiltonian by averaging with respect to the hole motion in the direction parallel to the quantum well growth axis. We have assumed parabolic band shape for conduction band. The system of non-homogeneous linear integro-differential equations is solved by expanding the solution in terms of eigenvectors of the Hamiltonian. Using the cylindrical symmetry approximation we are able to separate the angular variable and solve the resulting system of radial differential equations for the excitonic wave function numerically, using finite difference technique. Thus, contrary to more standard methods [3], we do not expand the radial part of the excitonic wave function into a set of localized functions and therefore we are able to cover the whole range of magnetic fields starting from zero field with relatively small number of mesh points. We have then fully taken into account the effects of band mixing due to the $k \cdot p$ coupling and Coulomb interaction as well as the symmetries of the optical matrix elements. 


\section{Results and discussion}

The photoluminescence excitation spectra have been taken on high quality $\mathrm{GaAs}-\mathrm{Ga}_{1-x} \mathrm{Al}_{x} \mathrm{As}$ single quantum wells of widths $40 \AA, 60 \AA$ and $80 \AA$ at $4 \mathrm{~K}$ for the magnetic fields $B$. up to $20 \mathrm{~T}$. The typical fan chart of the absorption peaks in $40 \AA$ thick quantum well is presented in Fig. 1. The lowest heavy-hole Landau level is clearly separated from the rest of the spectrum. In contrary, the excited levels intersect with light-hole exciton levels being subject to strong $k \cdot p$ mixing, especially in weaker magnetic fields. A very complicated shape of the higher-order part of the spectrum as well as heavily non-linear character of the lowest light-hole exciton Landau level are the effects of the band mixing. In particular, an avoided crossing between the lowest light-hole level and the second heavy-hole level can be observed at $B \approx 11 \mathrm{~T}$. The PLE spectrum displays three distinguishable maxima in the vicinity of the avoided crossing, suggesting that in fact three levels are optically active in this region.

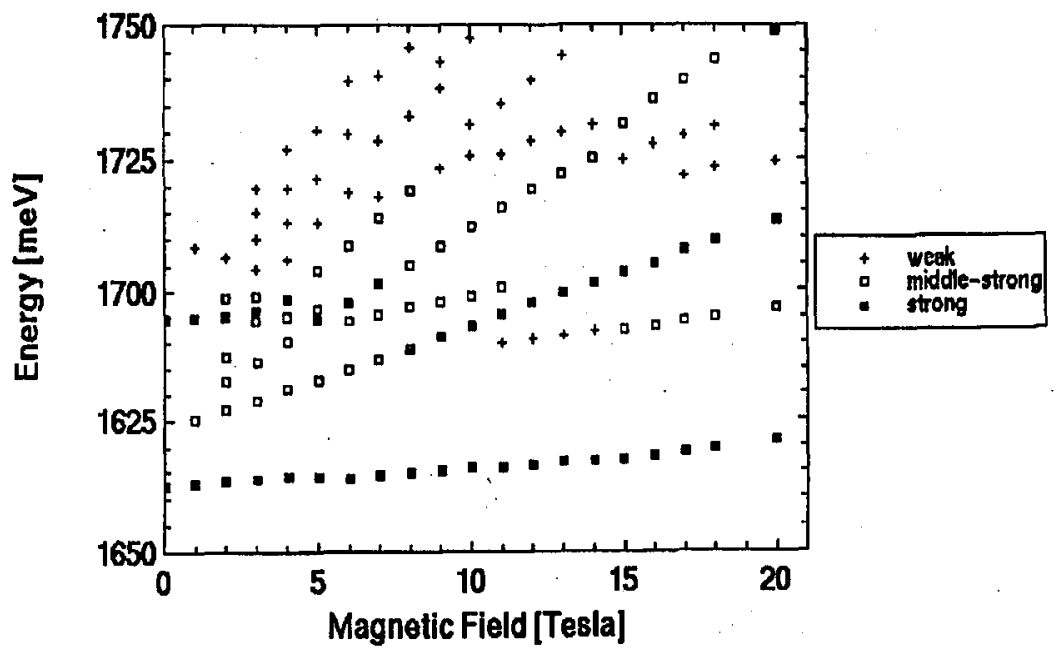

Fig. 1. Magnetic field dependence of the excitonic transition energies in $40 \AA$ quantum well for light polarization $\sigma^{-}$measured at $T=4 \mathrm{~K}$.

In our theoretical calculations we have used the barrier energy gap $E_{\mathrm{g}}$ and the biaxial strain $\epsilon_{x x}$ as fitting parameters. The best agreement with experiment has been achieved for $E_{\mathrm{g}}=2009 \mathrm{meV}, \epsilon_{x x}=0.0013$ (40 $\AA$ quantum well), $E_{\mathrm{g}}=$ $2004 \mathrm{meV}, \epsilon_{x x}=0.0006$ (60 $\AA$ quantum well) and $E_{\mathrm{g}}=2001 \mathrm{meV}, \epsilon_{x x}=0.0005$ ( $80 \AA$ quantum well), respectively. Small fluctuations of these parameters are probably within the experimental uncertainty.

In all calculations we have used the same values of the conduction and valence band offsets, $V_{c}=299 \mathrm{meV}, V_{v}=179 \mathrm{meV}$, respectively, although generally $E_{\mathrm{g}}, \epsilon_{\mathrm{xx}}, V_{\mathrm{c}}, V_{\mathrm{v}}$ should be fitted together. Fortunately, the relative distances between excitonic levels depend very weakly on $V_{\mathrm{c}}$ and $V_{\mathrm{v}}$. 
The quantum-well-region Luttinger constants and effective mass of electrons have been taken as $\gamma_{1}=6.79, \gamma_{2}=1.92, m_{\mathrm{e}}^{*}=0.0667$ in units of the free electron mass. The $g$-factors for the conduction and valence bands, $\kappa_{\mathrm{c}}=-0.42, \kappa_{\mathrm{v}}=1.2$, respectively, have been assumed not to vary from the barrier to the quantum-well material, in which case the envelope functions do not depend on the magnetic field. Similarly, the hydrostatic and shear deformation potentials, $a^{\mathrm{d}}=-8.7 \mathrm{eV}$, $b^{\mathrm{d}}=-1.8 \mathrm{eV}$ and elastic moduli, $C_{11}=11.9 \times 10^{11} \mathrm{dyn} / \mathrm{cm}^{2}, C_{12}=$ $5.38 \times 10^{11} \mathrm{dyn} / \mathrm{cm}^{2}$, have been treated as constants. The static dielectric constant has been taken as $\epsilon=13.18$.

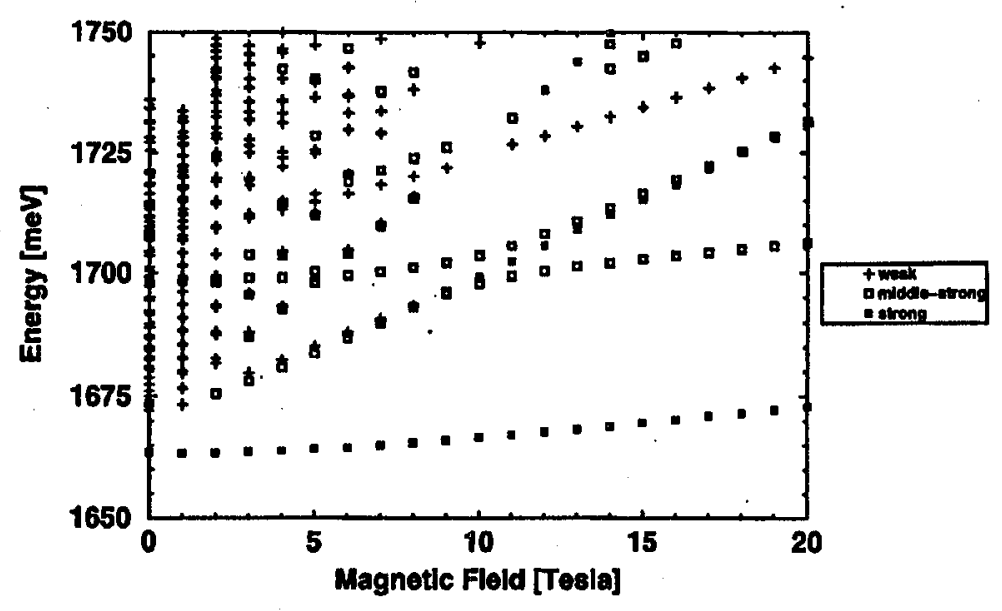

Fig. 2. Calculated magnetic field dependence of the excitonic transition energies in $40 \AA$ quantum well for light polarization $\sigma^{-}$.

The $k \cdot p$ mixing effects are clearly visible in our theoretical results presented in Fig. 2. Only a few lowest Landau levels are distinguishable and the excitonic spectrum at higher energies is very complicated. However, both lowest heavy-loole Landau level and the avoided crossing at $B=11 \mathrm{~T}$ are accurately reproduced.

It can be seen in Fig. 2 that in the region of weak magnetic fields the second heavy-hole exciton Landau level almost coincides with a weak, predominantly light-hole type exciton Landau level. These levels split at the magnetic field $B \approx 11 \mathrm{~T}$ : the lower one evolves into the light-hole level at high fields while the upper one transforms into the second heavy-hole level which merges with the lowest light-hole level at the magnetic field of about $12 \mathrm{~T}$. The presence of this additional light-hole level is induced by the $k \cdot p$ mixing between the light and heavy hole levels.

This effect was observed experimentally and qualitatively explained in [4]. Thus our model gives a quantitative confirmation of this phenomenon.

There are still some inaccuracies in our model which originate from two main sources.

First, in order to simplify our calculations, we have adopted a perfectly symmetric profile of the quantum-well potential, thus eliminating the interaction 
between hole subbands of different parity. Consequently, theoretical level splitting at the avoided crossing slightly underestimates the experimental value.

Next, we have neglected nonparabolicity of the conduction band, which causes the energies of absorption maxima to be overestimated, especially in stronger magnetic fields. This effect can be included in the theory at the level of derivation of the basic exciton equation. However, the resulting Hamiltonian is far more difficult for the computational purposes.

We can conclude that our model reproduces the main effects of $k \cdot p$ mixing on the magnetoabsorption spectra in quantum wells and our theoretical results are in considerably good correspondence with the experimental data. In particular, the predicted position of the fundamental heavy-hole spectral line and the avoided crossing between light and heavy Landau levels agree very well with the experimental data. Further improvement may be achieved by eliminating some approximations employed in our model.

\section{References}

[1] L. Viña, G.E.W. Bauer, M. Potemski, J.C. Maan, E.E. Mendez, W.I. Wang, Phys. Rev. B 41, 10767 (1990-II).

[2] W. Bardyszewski, D. Yevick, Phys. Rev. B 49, 5368 (1994-II).

[3] Ch. Stafford, S. Schmitt-Rink, W. Schaefer, Phys. Rev. B 41, 10000 (1990-I).

[4] M. Potemski, L. Viña, G.E.W. Bauer, J.C. Maan, K. Ploog, G. Weimann, Phys. Rev. B 43, 14707 (1991). 